\title{
The American Phytopathological Society Elected and Appointed Officers, Representatives, and Committees for 2002
}

Council

President: N. T. Keen

President-Elect: J. Fletcher

Immediate Past President: S. A. Slack

Vice President: G. C. Bergstrom

Secretary: C. A. Ishimaru

Treasurer: J. L. Sherwood

Editor-in-Chief, Phytopathology: B. I. Hillman

Editor-in-Chief, Plant Disease: A. R. Biggs

Editor-in-Chief, Molecular Plant-Microbe Interactions: H. P. Spaink

Editor-in-Chief, Phytopathology News: S. A. Johnston

Editor-in-Chief, APS Press: R. C. Ploetz

Senior Councilor-at-Large: S. S. Hurtt

Intermediate Councilor-at-Large: J. H. Andrews

Junior Councilor-at-Large: R. R. Belanger

Councilor of the Caribbean Division: S. Cantrell

Councilor of the North Central Division: F. W. Nutter Jr.

Councilor of the Northeastern Division: B. J. Christ

Councilor of the Pacific Division: M. E. Matheron

Councilor of the Potomac Division: A. Grybauskas

Councilor of the Southern Division: A. K. Culbreath

Ex officio: APS Foundation Representative: D. E. Mathre

Ex officio: Executive Vice President: S. C. Nelson

\section{Representatives}

American Association for the Advancement of Science: D. Gilchrist American Institute of Biological Sciences: G. C. Bergstrom

American Type Culture Collection: R. S. Nelson

Coalition for Funding Agricultural Research: S. A. Tolin

Coalition for Research on Plant Systems: S. A. Tolin \& J. L. Sherwood

Council for Agricultural Science and Technology: B. J. Jacobsen

International Society of Arboriculture: R. J. Stipes

International Society for Plant Pathology: Chair: A. M. Alvarez. G. S. Abawi, L.

V. Madden, J. Moyer, C. C. Mundt, B. W. Pennypacker, L. Sequeira, P. S. Teng,

A. H. van Bruggen, R. S. Zeigler

International Union of Microbiological Societies: C. J. Baker

Mycological Society of America: C. M. Stiles

National Committee on Worker Safety Standards: S. A. Alexander

National Council for Science and the Environment: T. A. Kucharek

National Research Council, Commission on Life Sciences: C. A. Ishimaru

National Research Council, CLS/Board of Agriculture: G. C. Bergstrom

Society of Environmental Toxicology and Chemistry: M. Simini

Society of Nematologists: E. L. Davis

APS Historian

P. D. Peterson, Jr.

APS Foundation, Board of Directors

Chair: D. E. Mathre. Vice Chair: B. J. Jacobsen. Past Chair: S. M. Coakley. Secretary-Treasurer: J. R. French. A. O. Paulus, D. A. Cooksey, P. R. Sellers, Ex officio: J. L. Sherwood, L. M. Sosnoskie, S. C. Nelson

Office of Electronic Communication (OEC) Board

Director: J. D. MacDonald. Director-Elect: J. B. Ristaino. C. T. Bull, A. O. Charkowski, D. M. Eastburn, G. G. Grove, N. Grunwald, G. Hughes, Ex officio: N. T. Keen

Office of International Programs (OIP) Advisory Board

Director: G. S. Abawi. Immediate Past Director: R. A. Bennett. L. L. Black, W. M. Brown, K. F. Cardwell, G. A. Forbes, E. R. French, J. H. McBeath, R. C. Ploetz, N. W. Schaad, J. R. Steadman, R. S. Zeigler, Ex officio: S. C. Nelson, A. O. Paulus

Education: Chair: K. F. Cardwell. J. McBeath, R. S. Zeigler

Policies and Program(s): Chair: H. J. Dubin. G. S. Abawi, L. Black, K. F. Cardwell, N. W. Schaad, J. R. Steadman

Research: Chair: N. W. Schaad. S. H. De Boer, G. A. Forbes, E. R. French

Service: Chair: J. R. Steadman. W. M. Brown, S. H. De Boer, T. A. Pastor-Corrales, R. C. Ploetz, R. Soufi

Publication no. P-2001-1126-010
Office of Industry Relations (OIR) Board

Director: C. M. Becker. Recording Director: M. R. Miles. R. P. Kaiser, J. L. Riggs, Z. Shi, M. Smither-Kopperl, M. G. Tiffany, Ex officio: M. N. Cline, C. L. Palmer Industry: Chair: K. W. Seebold, Jr. Immediate Past Chair: C. T. Schiller. Vice Chair: C. L. Palmer. D. T. Beadle, C. M. Becker, P. R. Haikal, G. M. Kemmitt, L. B. Laverdiere, C. S. Morton, M. R. Schwarz, L. E. Zang

Sustaining Associates: Chair: M. N. Cline. Members: TBD

Office of Public Affairs and Education (OPAE) Advisory Board

Director: G. L. Tylka. Immediate Past Director: G. C. Bergstrom. O. W. Barnett, M. N. Cline, C. R. Curtis, M. L. Daughtrey, M. A. Ellis, N. T. Keen, J. E. Partridge, J. B. Ristaino, J. R. Steadman

Public Policy Board (PPB)

Chair: O. W. Barnett. J. Amador, G. C. Bergstrom, N. B. Carroll, J. Fletcher, N. T. Keen, J. E. Leach, D. C. McGee, J. L. Sherwood, R. E. Stuckey, S. A. Tolin, M. Bjerkness, S. C. Nelson, G. L. Tylka

Scientific Programs Board

Director: E. N. Rosskopf. J. Fletcher, G. C. Bergstrom, S. S. Hurtt, R. M. Davis, G. L. Schumann

APS Publications Board

Chair: R. C. Ploetz. A. R. Biggs, B. I. Hillman, S. A. Johnston, J. D. MacDonald, T. Murray, H. P. Spaink, G. L. Schumann

Editors of APS Press

Editor-in-Chief: R. C. Ploetz. Senior Editors: C. M. Becker, D. M. Benson, A. Bergamin Filho, E. J. Braun, W. Chun, A. B. Gould, G. Hughes, J. B. Jones, G. W. Moorman, K.-B. G. Scholthof, Ex officio: J. L. Sherwood

APS Press Editorial Boards and Committees

Biological and Cultural Tests: Senior Editor: S. C. Bost. Section Editors: M. J. Boehm, K. L. Bowen, D. R. Cooley, G. J. Holmes, T. S. Isakeit, D. J. Jardine, A. P. Keinath, P. E. Lipps, G. P. Munkvold, J. Williams-Woodward

Fungicide and Nematicide Tests: Senior Editor: R. E. Baird. Section Editors: W. W. Bockus, P. Bristow, L. Datnoff, W. Elmer, A. Hagan, B. Padgett, R. N. Raid, D. Rosenberger, G. Schumann, D. Weingartner, K. Yoder, H. Young

Illustrations of Plant Pathogens and Diseases: Chair: Immediate Past Chair: E. J. Braun. G. J. Holmes, G. W. Hudler, V. Kumar, G. W. Moorman, N. R. O’Neill, J. E. Partridge, A. C. Schilder, G. R. Stanosz, T. J. Volk, J. E. Watkins

Phytopathological Classics: Chair: P. D. Peterson. Immediate Past Chair: T. D. Murray. D. W. Fulbright, C. S. Griffith, C. M. Liddell, K.-B. G. Scholthof

Standardization of Common Names of Plant Diseases: Chair: P. F. Bertrand. Vice Chair: TBD. Immediate Past Chair: M. Putnam. C. W. Averre, A. Grybauskas, J. H. Hart, M. E. Matheron, E. V. Podleckis, T. A. Zitter

Editors of MOLECULAR PLANT-MICROBE INTERACTIONS

Editor-in-Chief: H. P. Spaink. Senior Editors: C. A. Boucher, J. Glazebrook, F. Govers, D. Haas, M. J. Harrison, J. W. Kronstad, J. E. Schoelz, J. Stougaard, M. Udvardi, V. M. Williamson. Associate Editors: D. L. Beck, A. Becker, N. J. Brewin, J. P. Carr, Z. Chen, L. Ciuffetti, J. V. Cullimore, J. Dangl, M. Djordjevic, X. Dong, O. Geiger, G. Gheysen, S. E. Gold, M. Grant, S. Kamoun, B. N. Kunkel, K. Lambert, S. A. Lommel, T. McDermott, J. McDowell, Y. MoenneLoccoz, P. Müller, D. Noel, T. Nuernberger, M. Parniske, K. Pawlowski, S. Perotto, J. M. Salmeron, F. Sanchez, H. B. Scholthof, S. C. Somerville, J. Sweigard, K. Szczyglowski, N. J. Talbot, I. K. Toth, J. Van Kan, N. D. Young

\section{Editors of PHYTOPATHOLOGY}

Editor-in-Chief: B. I. Hillman. Senior Editors: D. M. Bird, D. A. Cuppels, D. R. Fravel, R. L. Gilbertson, S. H. Hulbert, R. S. Nelson, K. V. Subbarao, X.-B. Yang, C. C. Mundt. Associate Editors: M. K. Clayton, J. C. Correll, J. N. Culver, C. M. Deom, J. A. Duthie, C. M. Fauquet, R. D. Frederick, S. E. Gold, T. R. Gordon, N. H. Grimsley, R. W. Jones, D. Y. Kobayashi, F. N. Martin, R. R. Martin, P. V. Oudemans, D. G. Panaccione, T. L. Peever, D. Rigling, M. J. Roossinck, A. Rowhani, C. L. Schardl, H. Scherm, D. A. Schisler, S. M. Schneider, J. E. Sherwood, D. C. Stenger, V. O. Stockwell, F. van den Bosch, W. M. Wintermantle, C.-L. Xiao, X. Xu

\section{Editors of PLANT DISEASE}

Editor-in-Chief: A. R. Biggs. Senior Editors: K. L. Bowen, B. D. Bruton, P. D. Colyer, L. L. Domier, T. C. Harrington, M. K. Hausbeck, P. J. Landschoot, C.-H. Liao, G. P. Munkvold, D. L. Seifers, K. L. Stevenson. Feature Editor: N. A. Tisserat. Disease Notes Assigning Editor: A. P. Keinath. Focus Editor: T. 
Kommedahl. Associate Editors: J. O. Becker, P. Bonello, B. M. Cunfer, L. J. du Toit, K. C. Eastwell, F. A. Gray, H. M. Griffiths, R. W. Hammond, J. R. Hartman, D. E. Hershman, R. M. Hunger, L. L. Kinkel, G. Kuldau, J. S. Lehman, D. S. Marshall, J. L. McEvoy, T. E. Mirkov, G. Newcombe, J. W. Pscheidt, P. L. Pusey, J. S. Russin, D. A. Samac, L. W. Timmer, J. Y. Uchida, G. Y. Yuen

\section{Editors of PLANT HEALTH PROGRESS}

Editor-in-Chief: T. Murray. Senior Editors: A. M. Alvarez, T. J. Gerik, G. G. Grove, K. Moore, H. V. Morton, S. T. Nameth, M. Putnam, K. K. Rane, J. L. Starr, K. L. Steffey, Ex officio: A. P. Keinath, R. C. Ploetz, J. B. Ristaino

\section{Editors of THE PLANT HEALTH INSTRUCTOR}

Editor-in-Chief: G. L. Schumann. M. L. Agnew, M. J. Boehm, C. J. D’Arcy, D. M. Eastburn, D. C. Gross, K. B. Johnson, M. L. Powelson, D. L. Schadler, D. O. TeBeest

Phytopathology News Advisory: Chair: S. A. Johnston, J. H. Andrews, A. Grybauskas, D. M. Rizzo

\section{Society Internal Relations}

Awards and Honors: Chair: G. A. Payne. Vice Chair: L. D. Dunkle. Immediate Past Chair: W. R. Stevenson. T. J. Burr, D. C. Gross, M. L. Powelson, R. C. Rowe Committee on Committees: Chair: S. S. Hurtt, J. H. Andrews, R. R. Belanger

Financial Advisory: Chair: J. L. Sherwood. J. H. Andrews, G. C. Bergstrom, J. Fletcher, N. T. Keen, M. E. Matheron, R. C. Ploetz

Headquarters Operations: Chair: J. N. BeMiller, J. L. Sherwood, J. Fletcher, N. T. Keen, AACC representatives, Ex officio: S. C. Nelson

Placement: Chair: S. L. Walker. Vice Chair: TBD. Immediate Past Chair: E. A. Sobek. C. H. Bock, A. K. Culbreath, R. Rong, A. K. Wood

\section{Society General Policies}

Biotechnology Impact Assessment: Chair: R. R. Martin. Vice Chair: D. C. Sands. Immediate Past Chair: B. K. Duffy. J. H. Andrews, A. O. Charkowski, L. G. Kovacs, V. Kumar, B. Meng, S. F. Shamoun, P. Warrior, Y. Yang

Collections and Germplasm: Chair: M. E. Miller. Vice Chair: J. P. Wilson. Immediate Past Chair: J. C. Rupe. M. L. Carson, A. G. Gillaspie, Jr., B. Lovic, E. A. Milus, D. V. Phillips, R. W. Schneider, H. F. Schwartz

Cultural Diversity: Chair: R. L. Grayson. Vice Chair: TBD. Immediate Past Chair: C. M. Okpodu. M. T. Abdullah, D. G. Fernando, D. M. Huber, T. L. Kirkpatrick, B. P. Singh, M. Skaria, R. H. von Qualen

Extension: Chair: D. E. Legard. Vice Chair: W. W. Turacheck. Immediate Past Chair: M. A. Draper. G. S. Bender, E. A. Brown, II, R. M. Davis, E. D. De Wolf, R. L. Forster, T. S. Isakeit, S. G. P. Nameth, M. A. Newman, L. P. Pottorf, R. L. Schlub

Graduate Student: Chair: L. M. Sosnoskie. Vice Chair: Z. Kanaan-Atallah. Immediate Past Chair: A. E. Glenn. R. Bulluck, J. M. Clifford, L. E. Davidson, P. D. Esker, E. M. Martin, P. R. Northover, M. A. Patil, L. D. Price, J. D. Reddy, S. J. Scheuerell, P. M. Sforza, P. Siddavatam, S. Thomas, L. P. Tredway, D. I. Tsitsigiannis

Private Practice: Chair: W. T. Cobb. Vice Chair: B. Rao. Immediate Past Chair: H. C. Mellinger. J. H. Hart, W. R. Landis, H. D. Quemada, G. A. Snow

Regulatory Plant Pathology: Chair: S. D. Cohen. Vice Chair: K. L. Kosta. Immediate Past Chair: L. G. Brown. T. N. Boratynski, A. J. Caesar, T. R. Gottwald, G. Hughes, P. I. Lewis, G. M. Milbrath, C. M. Ocamb, D. C. Opgenorth, G. L. Peterson, S. A. Rizvi, S. R. Shafer, A. T. Tschanz

Teaching: Chair: M. A. Sulzinski. Vice Chair: M. A. Langham. Immediate Past Chair: M. B. Riley. A. B. Baudoin, E. J. Braun, M. J. Clements, L. Z. De Luna, J. C. Locke, K. S. McLean, C. S. Rothrock, P. Vincelli

Women in Plant Pathology: Chair: P. D. Adams. Vice Chair: TBD. Immediate Past Chair: L. E. Hanson. M. T. Abdullah, S. M. Bissonnette, S. C. Butterworth, S. Carle, M. K. Hausbeck, C. Loschinkohl, L. Ortiz-Ribbing, E. Rosskopf, M. Smitter-Kopperl, N. K. Zidack

Youth Program: Chair: K. Shelton. Vice Chair: C. A. Jasalavich. Immediate Past Chair: R. N. Raid. Y. T. Berthier-Schaad, J. J. Choi, L. D. Moore, B. L. RandallSchadel

\section{Subject Matter}

Bacteriology: Chair: F. J. Louws. Vice Chair: S. M. Brumbley. J. D. Barak, G. A. Beattie, C. T. Bull, J. S. Hartung, S. Y. He, E. N. Okey, S. Q. Pan, L. S. Pierson, III, B. K. Scholz-Schroeder, K. L. Schroeder

Biochemistry, Physiology, and Molecular Biology: Chair: T. J. Wolpert. Vice Chair: A. F. Bent. Immediate Past Chair: R. G. Upchurch. J. P. Bolar, A. M. Fakhoury, T. K. Mitchell, C. M. Okpodu, L. D. Price, R. Rong, S. B. Von Bodman, Y. Yang

Biological Control: Chair: W. F. Stoneman. Vice Chair: B. B. McSpaddenGardener. Immediate Past Chair: C. M. Press. S. M. Boyetchko, L. D. Butler, A. J. Caesar, B. K. Duffy, N. I. Kahn, B. H. Ownley, E. N. Rosskopf, Y. M. Shabana, K. R. Vanga, R. Vargas, P. Warrior
Chemical Control: Chair: D. B. Langston. Vice Chair: P. M. Brannen. Immediate Past Chair: N. Lalancette. R. D. Cartwright, J. E. Fajardo, R. E. Gold, R. C. Kemerait, C. L. Kohls, T. M. Momol, D. E. Wedge, F. P. Wong

Diagnostics: Chair: J. W. Beale. Vice Chair: L. J. Du Toit. Immediate Past Chair: K. K. Rane. B. D. Ambruzs, M. Babadoost, E. M. Bentley, J. H. Brock, T. C. Creswell, G. G. Grove, J. Mullen, M. Putnam, D. L. Scott, K. L. Snover

Diseases of Ornamental Plants: Chair: G. A. Chastagner. Vice Chair: J. W. Buck. Immediate Past Chair: A. B. Sinderman. A. R. Chase, R. A. Cloyd, M. R. Corral Garcia, J. H. Dobbs, A. B. Gould, J. L. Hall, M. K. Hausbeck, C. Hong, J. C. Locke, G. W. Moorman

Environmental Quality and Plant Health: Chair: C. R. Krause. Vice Chair: S. R. Shafer. Immediate Past Chair: P. A. Gay. J. A. Chavarria, C. R. Curtis, C. L. Lennox, T. S. Schubert, M. Simini, G. S. Smith, S. R. Vann

Epidemiology: Chair: K. A. Garrett. Vice Chair: S. J. Pethybridge. Immediate Past Chair: W. F. Pfender. S. Chakraborty, E. D. De Wolf, D. G. Fernando, G. A. Forbes, J. Hao, J. J. Marois, A. L. Mila, F. W. Nutter, P. V. Oudemans, H. $\mathrm{Su}, \mathrm{B} . \mathrm{M} . \mathrm{Wu}$

Forest Pathology: Chair: L. M. Haugen. Vice Chair: G. R. Stanosz. Immediate Past Chair: W. J. Otrosina. P. Bonello, J. N. Bruhn, M. M. Garbelotto, R. S. Hunt, J. T. Kliejunas, N. B. Klopfenstein, J. G. O’Brien, P. J. Zambino

Genetics: Chair: S. E. Gold. Vice Chair: S. Kang. Immediate Past Chair: T. L. Peever. R. G. Bhat, G. W. Douhan, A. E. Glenn, Y. Jin, S. Kamoun, G. Kuldau, J. S. Lehman, C. M. Liddell, G.-L. Wang

Host Resistance: Chair: A. E. Dorrance. Vice Chair: J. A. Kolmer. Immediate Past Chair: J. A. Thies. X. Chen, M. J. Clements, K. L. Crabtree, P. T. Himmel, C. S. Kousik, F. N. Lee, H. K. Ngugi, J. L. Norelli, B. A. Perez, M. G. Redinbaugh, R. W. Schneider, C. E. Thomas, G. L. Tylka, I. E. Yates

Integrated Pest Management: Chair: S. A. Alexander. Vice Chair: L. Fought. Immediate Past Chair: G. L. Schuster. W. M. Brown, Jr., A. K. Culbreath, D. S. Egel, S. A. Heuchelin, K. L. Pernezny, J. L. Riggs, H. Scherm, S. F. Shamoun, E. J. Sikora, G. S. Smith, N. Zriba

Mycology: Chair: C. M. Stiles. Vice Chair: W. Chen. Immediate Past Chair: M. A. Cubeta, R. E. Baird, M. L. Boyd, D. B. Chalkey, J. C. Correll, S. Demir, D. M. Eastburn, S. B. Goodwin, D. J. Royse, L. Wasilwa

Mycotoxicology: Chair: G. P. Munkvold. Vice Chair: A. E. Desjardins. H. K. Abbas, K. E. Damann, R. Dill-Macky, G. A. Gilbert, N. J. P. Keller, G. N. Odvody, D. G. Panaccione, A. Schaafsma, D. A. Schisler, D. I. Tsitsigiannis, G. L. Windham

Nematology: Chair: W. T. Crow. Vice Chair: TBD. Immediate Past Chair: C. S. Johnson. S. Chen, R. F. Davis, D. W. Dickson, J. Esnard, T. L. Kirkpatrick, S. A. Lewis, A. E. MacGuidwin, J. P. McMahon

Pathogen Resistance: Chair: T. B. Brenneman. Vice Chair: H. L. Ypema. Immediate Past Chair: P. I. Lewis. T. Chand-Goyal, K. L. Crabtree, A. M. Diner, S. N. Jeffers, P. S. McManus, R. A. Romero, S. M. Schneider, K. L. Stevenson, J. A. Yocum,

Phyllosphere Microbiology: Chair: C. C. Mundt. Vice Chair: S. Lindow. O. Carisse, V. J. Elliott, B. J. Jacobsen, K. B. Johnson, O. Kilic, W. F. Mahafee, J. Mercier, P. L. Pusey, B. D. Shaw, G. Y. Yuen

Plant Pathogen and Disease Detection: Chair: C. A. Levesque. Vice Chair: TBD. Immediate Past Chair: L. E. Levy. J. J. Beck, R. M. DeVries-Paterson, N. Glynn, N. L. Robertson, W. H. Sheta, M. A. Sulzinski, E. Vivoda, J. Q. Xia

Plant Disease Losses: Chair: W. W. Turechek. Vice Chair: S. E. Penix Fitzsimmons. Immediate Past Chair: S. Chakraborty. J. C. Comstock, J. Guan, C. A. Hollier, G. Hughes, J. E. Lundquist, L. V. Madden, M. H. Royer

Postharvest Pathology: Chair: W. S. Conway. Vice Chair: W. J. Janisiewicz. J. E. Adaskeveg, E. M. Imai, D. F. Kendra, J. L. McEvoy, T. J. Michailides, D. Prusky, P. G. Sanderson, J. L. Smilanick, D. Sugar, J. Zhang

Seed Pathology: Chair: C. J. Kurowski. Vice Chair: L. M. Shepherd. C. C. Block, P. Fenn, S. M. Koehler, T. A. Kucharek, M. E. Meadows, B. L. RandallSchadel, A. B. Thornton, M. G. Tiffany, R. R. Walcott, W. L. Wiebe

Soil Microbiology and Root Diseases: Chair: M. L. Smither-Kopperl. Vice Chair: J. H. Bowers. Immediate Past Chair: D. R. Fravel. R. Bulluck, C. H. Canady, C. G. Eayre, W. H. Elmer, M. S. Krause, J. E. Kurle, P. Lemanceau, R. J. McGovern, S. L. Meyer, J. L. Parke, J. M. Raaijmakers, M. S. Reddy, K. G. Shetty, A. G. Stone

Tropical Plant Pathology: Chair: J. V. Da Graca. Vice Chair: S. S. Savary. Immediate Past Chair: J. Y. Uchida. R. Bandyopadhyay, L. A. Calvert, D. R. Greenough, M. P. Grisham, N. A. Harrison, S. Kelemu, D. N. Kuhn, R. G. McGuire, J. J. Ooka, R. P. Rodriguez, A. Westphal

Turfgrass Pathology: Chair: L. J. Stowell. Vice Chair: J. F. Powell. Immediate Past Chair: M. J. Boehm. M. L. Agnew, L. E. Datnoff, S. P. Falk, K. J. Gard, D. Y. Han, G. Jung, R. X. Latin, S. B. Martin, E. A. Milus, B. Rao, M. R. Schwarz, W. Uddin, J. M. Vargas, N. R. Walker

Virology: Chair: J. F. Murphy. Vice Chair: J. E. Schoelz. Immediate Past Chair: W. M. Wintermantel. S. T. Adkins, J. M. Anderson, V. Bowman-Vance, R. H. Brlansky, A. L. Eggenberger, A. V. Karasev, R. C. Larsen, H. Liu, G. Loebenstein, C. J. M. Maroon, B. Meng, J. E. Polston, W. Qiu, B. R. Reddick, J. Verchot Lubicz 